\title{
Duodenal-jejunal bypass liner implantation provokes rapid weight loss and improved glycemic control, accompanied by elevated fasting ghrelin levels
}

\author{
Authors \\ Parweez Koehestanie ${ }^{2}$, Kemal Dogan ${ }^{2}$, Frits Berends ${ }^{2}$, Ignace Janssen², Peter Wahab ${ }^{3}$, Marcel Groenen ${ }^{3}$, \\ Michael Müller ${ }^{1}$, Nicole de Wit ${ }^{1}$ \\ Institutions \\ Division of Human Nutrition, Wageningen University, Wageningen, The Netherlands \\ ${ }^{2}$ Department of General Surgery, Rijnstate, Arnhem, The Netherlands \\ ${ }^{3}$ Department of Gastroenterology, Rijnstate, Arnhem, The Netherlands
}

received

20. August 2013

accepted after revision

8. January 2014

\section{Bibliography}

DOI http://dx.doi.org/

10.1055/s-0034-1365222

Published online: 7.3.2014

Endoscopy International Open

2014; 02: E21-E27

(c) Georg Thieme Verlag KG

Stuttgart · New York

E-ISSN 2196-9736

\section{Corresponding author}

\section{Nicole de Wit, PhD}

Human Nutrition, Wageningen University

Bomenweg 2

Wageningen $6703 \mathrm{HD}$

The Netherlands

nicole.dewit@wur.nl
Background and study aims: Endoscopic implantation of a duodenal-jejunal bypass liner (DJBL) is a novel bariatric technique to induce weight loss and remission of type 2 diabetes mellitus. Placement of the DJBL mimics the bypass component of the Roux-en-Y gastric bypass (RYGB) procedure. In this observational study, we evaluated improvement of glycemic control and weight loss in the course of the treatment $(0-24$ weeks after DJBL implantation) and analyzed accompanying gut hormone responses.

Patients and methods: 12 obese individuals with type 2 diabetes were selected for DJBL implantation. Body weight, fat mass, and fasting plasma levels of glucose, insulin, C-peptide, and glycated hemoglobin (HbA1c), were analyzed at $0,1,4$ and 24 weeks post-implant. Fasting ghrelin, gastric inhibitory peptide (GIP), and glucagon-like peptide (GLP-1) were determined at 0,1 and 4 weeks post-implant.

\section{Introduction \\ $\nabla$}

Overweight and obesity are major risk factors for several chronic diseases, including diabetes, cardiovascular diseases, and cancer [1]. Once considered a problem only in economically developed countries, overweight and obesity are now dramatically on the rise in economically developing countries, particularly in urban settings [2]. It is well established that obesity promotes insulin resistance and in doing so, forms the most important risk factor in developing type 2 diabetes. Today more than 60 million people worldwide face the dual challenge of managing type 2 diabetes and obesity [2,3].

Weight loss improves insulin resistance and next to medication is one of the most important treatment modalities. Although conservative therapy such as diets and lifestyle training are frequently
Results: Besides significant weight loss, fat mass, fasting insulin, and homeostasis model assessment-estimated insulin resistance (HOMA-IR) index were also significantly decreased after DJBL implantation and a $42 \%$ reduction was found in diabetes medication $(P<0.05)$. The fasting GLP-1 response in the first 4 weeks post-implant was significantly correlated with the fasting insulin and HOMA-IR response. Fasting ghrelin was found to be significantly elevated, in contrast to the decrease in ghrelin that is found after RYGB surgery.

Conclusions: DJBL implantation provoked significant weight loss, a decrease in fat mass, and an early remission of type 2 diabetes, comparable to results seen after RYGB surgery. Gut hormone analyses revealed a potential role of fasting GLP1 in early remission of type 2 diabetes. Interestingly, the DJBL-induced elevation of ghrelin contradicts the suggested role of reduced ghrelin levels after RYGB in improvement of glycemic control.

successful in weight control in the short term, long-term results are most often disappointing because of problems with compliance with diets and lifestyle changes [4]. Bariatric surgery, on the other hand, has proven its effectiveness in achieving and maintaining weight loss and improving obesity-related type 2 diabetes, quality of life, and survival [5]. It has been recognized that Roux-en-Y gastric bypass (RYGB) surgery causes remission of type 2 diabetes in the majority of patients within days after the surgery [6,7]. This suggests that mechanisms that are independent of weight loss are responsible for the early remission. It has been proposed that the early improvement in glycemic control following RYGB partly depends on the changes in intestinal anatomy and circulating levels of gut hormones, especially ghrelin and the incretin hormones, gastric inhibitory peptide (GIP) and glucagon-like peptide-1 
(GLP-1) $[8,9]$. Ghrelin and GIP are assumed to be main effectors in the "foregut hypothesis" that states that exclusion of the foregut from nutrient exposure is the factor most responsible for the rapid improvement of hyperglycemia $[10,11]$. GLP-1 is the main candidate for the "hindgut theory" that explains improved glycemia by a faster delivery of nutrients to the hindgut after RYGB surgery. Both theories are currently still debated 12. Although RYGB surgery is an efficient bariatric intervention for remission of type 2 diabetes, this surgery is a rather drastic measure that not every patient or doctor would prefer as a first treatment. Among the reasons not to embark on bariatric surgery are that body mass index (BMI) is less than $35 \mathrm{~kg} / \mathrm{m}^{2}$ and thus does not reach National Institutes of Health (NIH) criteria for this procedure, and fear of surgery and/or its complications.

Recently, a novel nonsurgical bariatric technique has been developed using the duodenal-jejunal bypass liner (DJBL). The DJBL consists of a $60 \mathrm{~cm}$ long fluoropolymer sleeve which can be delivered into and retrieved from the duodenum endoscopically. Once placed in the duodenum it mimics the intestinal bypass component of the RYGB. Clinical experience to date in more than 600 patients has demonstrated its safety and efficacy and the ability of the DJBL to rapidly affect blood glucose control and weight loss, while also demonstrating improvements in metabolic functions over time, including in cholesterol, blood pressure, low density lipoprotein (LDL), and triglycerides levels [13-16].

Our goal was to investigate the effect of the DJBL on body weight, fat mass, and early remission of type 2 diabetes during 6 months after implantation and to explore the potential role of gut hormones in DJBL-induced effects on weight loss and improvement of glycemic control. Therefore, body weight, fat mass, and fasting plasma levels of glucose, insulin, C-peptide, and HbA1c were analyzed pre-implantation and after 1, 4 and 24 weeks post-implant. Additionally, at the first three time points, fasting plasma levels of ghrelin, GIP, and GLP-1 were determined.

\section{Patients and methods}

$\nabla$

The study was performed according to the principles of the declaration of Helsinki. The study was approved by the research and ethics committee at Rijnstate Hospital Arnhem, the Netherlands (protocol number: 1141), functioning according to the 3 rd edition of the Guidelines on the practice of ethics committees in medical research issued by the Royal College of Physicians of London. Written informed consent was obtained from all participants after full explanation of the purpose and nature of all procedures.

\section{Patients}

Patients were considered eligible for the study if they were between 18 and 60 years old; had a BMI between 28 and $35 \mathrm{~kg} / \mathrm{m}^{2}$; and type 2 diabetes with an HbA1c level above $7 \%$. Patients were allowed to take metformin, sulfonylurea derivates and/or insulin. Exclusion criteria were: pregnancy or intention to become pregnant; use of nonsteroidal anti-inflammatory drugs (NSAIDS), anticoagulation therapy, corticosteroids, weight loss medication, or drugs known to affect gastrointestinal motility; substance abuse; active Helicobacter pylori infection; dysfunctional $\beta$ cells (C-peptide of $<0.75 \mathrm{nmol} / \mathrm{L}$ ); iron deficiency or iron deficiency anemia; gastrointestinal tract abnormalities or previous surgery in the gastrointestinal tract that could affect the ability to place the device; symptomatic gallstones or kidney stones; known infection; bleeding disorders; gastroesophageal reflux disorder; connective tissue disorders; or severe liver or kidney failure (creatinine $>180$ $\mathrm{mmol} / \mathrm{L})$.

\section{Study protocol}

For this observational study, 12 participants ( 7 men, 5 women) who were obese (BMI $33.5 \pm 0.8 \mathrm{~kg} / \mathrm{m}^{2}$ ) and had type 2 diabetes (duration of type 2 diabetes $7.4 \pm 1.4$ years) were selected for implantation of a DJBL and followed during 24 weeks post-implantation. During this study, body weight and fat mass were assessed using a body composition analyzer.

Before implantation of the DJBL and 1, 4 and 24 weeks post-implantation of the DJBL, blood was drawn in ethylenediaminetetraacetic (EDTA) tubes (Greiner Bio-One B.V, Alphen a/d Rijn, The Netherlands) after an overnight fasting period (at least 12 hours). Fasting plasma levels of gut hormones were only analyzed pre-implantation and at 1 and 4 weeks post-implantation. Within seconds after blood withdrawal (at 0, 1 and 4 weeks after DJBL implantation) 10 microliter per millilitre of blood dipeptidyl peptidase 4 (DPP4) inhibitor (Merck Millipore, Billerica, MA, USA) was added to prevent degradation of active GLP-1 and GIP. Blood was centrifuged (within 1 hour) at $1000 \times$ g for 10 minutes at $4{ }^{\circ} \mathrm{C}$. Plasma was then aliquoted, snap-frozen and stored at $-80^{\circ} \mathrm{C}$. Glucose, HbA1c, and C-peptide levels were measured at Rijnstate Hospital Arnhem following a standardized clinical protocol. The medication score was based on medication use for hyperglycemia control and was assessed before and within the first week after DJBL implantation. It was based on the scoring system reported by Dorman et al. [17] (i.e., one point for each oral diabetes medication, one point for exenatide injections, two points for insulin injections) in combination with a scoring system in which for each medication unit one additional point was added (medication units: metformin $500 \mathrm{mg}$, glimepiride $2 \mathrm{mg}$, tolbutamide $500 \mathrm{mg}$, vildagliptin $50 \mathrm{mg}$, insulin glargine $10 \mathrm{eh}$, insulin aspart $10 \mathrm{eh}$, exenatide $10 \mathrm{eh}$ [eh = units]).

\section{Gut hormone assays}

Commercially available enzyme-linked immunosorbent assays (ELISAs) were used according to the manufacturer's protocol to measure total ghrelin, active GLP-1, and total GIP concentrations (EZGRT-89K, EGLP-35K, EZHGIP-54K, respectively; Merck Millipore, Billerica, MA, USA) in human EDTA plasma samples at 0, 1 and 4 weeks after DJBL implantation.

\section{DJBL procedure}

The DJBL (Endobarrier, GI Dynamics, Lexington, MA, USA) is an endoscopic implant that mimics the intestinal bypass component of the RYGB ( $\bullet$ Fig.1 a,b). The device comprises a $60 \mathrm{~cm}$ long impermeable fluoropolymer liner and a nitinol anchor, which is used to reversibly affix the device to the wall of the duodenum ( $\bullet$ Fig.1c). The anchor is located in the duodenal bulb and the liner stretches out through the duodenum and into the jejunum. The DJBL is open at both ends to allow food passage. As a result, food will pass through the interior of the DJBL while pancreatic and bile juices stay on the outside of the liner, so that digestion and absorption of nutrients can only start at the end of the liner. The DJBL therefore creates a bypass of the proximal intestinal tract.

Implantation of the DJBL was performed with the patient under conscious sedation with propofol. Initial access to the stomach and duodenum was achieved by a standard gastroduodenoscopy. Next, a guidewire was advanced into the duodenum and the encapsulated device on a custom catheter was tracked over the 


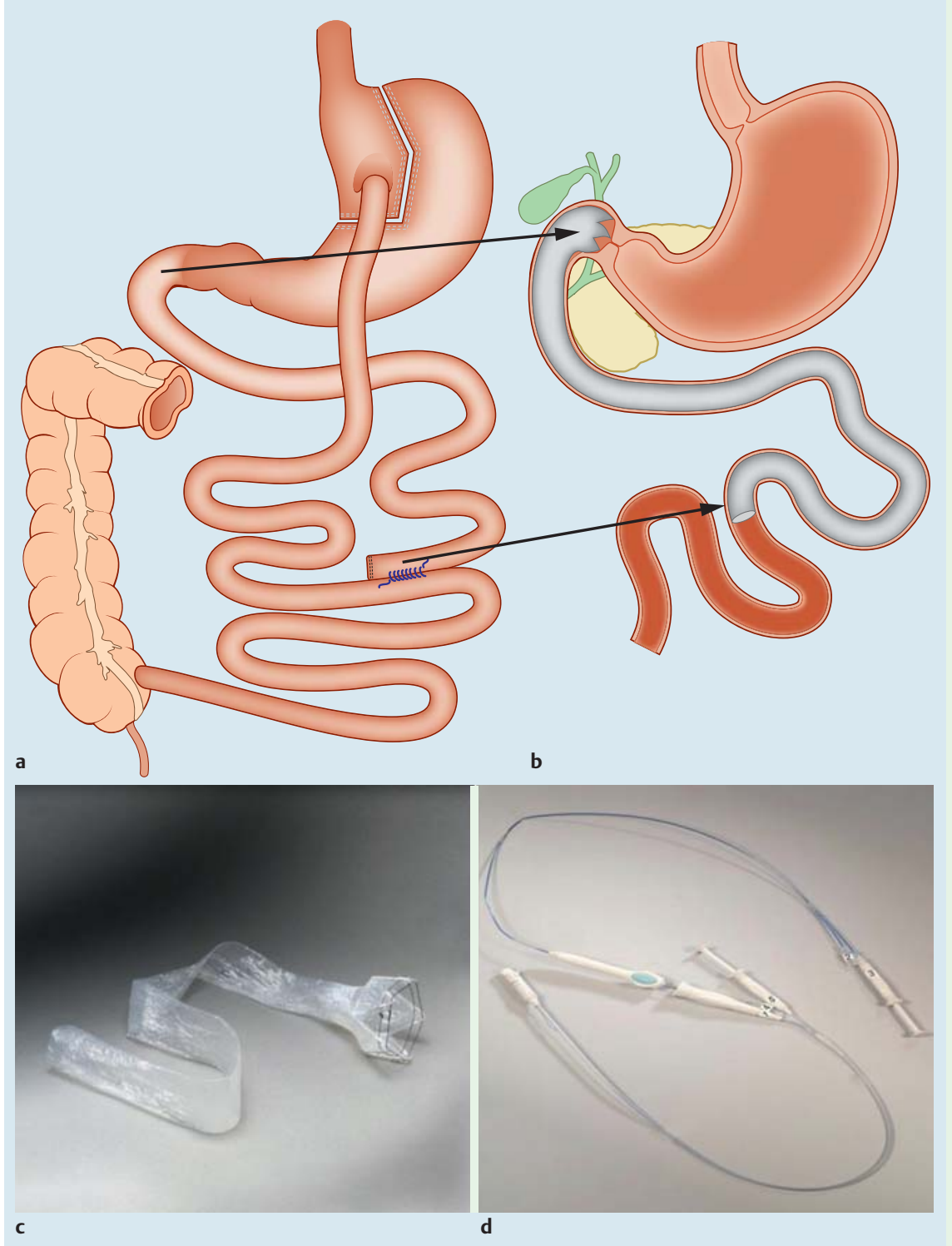

Fig. 1 Implantation of duodenal-jejunal bypass liner (DJBL). a, b Schematic overview of Roux-en-Y gastric bypass (RYGB) surgery (a) and implantation of DJBL (b). The arrows designate the comparable duodenal-jejunal part of the intestine that is "bypassed" in both bariatric procedures. c The DJBL: $60 \mathrm{~cm}$ long impermeable fluoropolymer sleeve and a nitinol anchor. $\mathbf{d}$ DJBL implantation system.

guidewire into the duodenum ( $\mathbf{F i g} .1 \mathrm{~d}$ ). The capsule at the distal end holds the liner and anchor. The catheter has an atraumatic ball at the end which was advanced through the intestine deploying the liner behind it. After full extension of the liner, the anchor was deployed in the duodenal bulb, approximately $0.5 \mathrm{~cm}$ distal to the pylorus. Endoscopic and fluoroscopic guidance was used to verify the correct positioning of the DJBL. The DJBL was removed after 24 weeks.

After placement of the DJBL, patients received dietary guidelines including a low calorie diet (female $1200 \mathrm{kcal}$, male $1500 \mathrm{kcal}$ ). Moreover, patients were advised to consume only liquids (e.g., water, tea, bouillon, milk, soup, yogurt drinks) and pureed fruit in the first 3 days post-implant and soft, moist, ground, or puréed foods (e.g., yogurt, diced vegetables and fruit, smoothie, rice pudding, scrambled egg) on days $4-7$ after DJBL implantation, after which they could resume a normal diet. Our nutritionists contacted the patients before and 1, 4, 12 and 24 weeks after DJBL implantation to check and stimulate compliance with the dietary guidelines.

\section{Statistical analysis}

Data are reported as the mean \pm standard error (SE). The differences between the mean values were tested for statistical significance using a one-way analysis of variance (ANOVA) with least significant difference (LSD) post hoc test or paired samples $t$ test. A Pearson correlation analysis was performed using area under the curve (AUC) data of $0-4$ weeks after DJBL implantation or 0-24 weeks post-implant. PASW Statistics 19.0 software was used (SPSS Inc., Chicago, Illinois, USA).

\section{Results}

\section{Effect of DJBL on weight loss and glycemic control}

A total of 12 obese patients (BMI $\geq 30 \mathrm{~kg} / \mathrm{m}^{2}$ ) with type 2 diabetes, having an average age of $50.3 \pm 1.9$ years, were selected for implantation of a DJBL. No complications due to implantation of the DJBL were reported and therefore none of the patients had to undergo removal of the DJBL before the 24-week time point. In these patients, placement of the DJBL accompanied by the help of dietary guidelines resulted in a significant weight loss ( Table 1 and $\bullet$ Fig.2). This weight loss was already manifest 
Table 1 Patient $(n=12)$ characteristics before and after implantation of duodenal-jejunal bypass liner (DJBL).

\begin{tabular}{|c|c|c|c|c|c|c|c|c|c|}
\hline & \multicolumn{8}{|c|}{ Weeks post-implant } & \multirow[t]{3}{*}{$P$ value } \\
\hline & \multicolumn{2}{|l|}{0} & \multicolumn{2}{|l|}{1} & \multicolumn{2}{|l|}{4} & \multicolumn{2}{|l|}{24} & \\
\hline & mean & SE & mean & SE & mean & SE & mean & SE & \\
\hline Body weight, kg & 104.9 & 3.0 & 101.3 & 2.9 & 99.9 & 2.9 & 97.7 & 3.3 & 0.39 \\
\hline Weight loss, kg & $0.0^{\mathrm{a}}$ & 0.0 & $-3.6^{b}$ & 0.5 & $-5.1^{b}$ & 0.9 & $-7.2^{\mathrm{b}}$ & 1.2 & $<0.05$ \\
\hline $\mathrm{BMI}, \mathrm{kg} / \mathrm{m} 2$ & 33.5 & 0.8 & 32.3 & 0.8 & 31.9 & 0.8 & 31.2 & 1.0 & 0.24 \\
\hline Fat mass, \% & $40.3^{\mathrm{a}}$ & 1.7 & $40.0^{\mathrm{a}}$ & 1.9 & $35.0^{\mathrm{a}, \mathrm{b}}$ & 1.9 & $33.1^{\mathrm{b}}$ & 1.8 & $<0.05$ \\
\hline $\begin{array}{l}\text { Fasting glucose, } \\
\mathrm{mmol} / \mathrm{L}\end{array}$ & 12.1 & 0.7 & 9.7 & 1.2 & $9.5^{2}$ & 0.3 & 10.6 & 0.7 & 0.21 \\
\hline Fasting insulin, $\mathrm{mU} / \mathrm{L}$ & $21.5^{\mathrm{a}}$ & 6.0 & $11.4^{\mathrm{a}, \mathrm{b}}$ & 2.5 & $7.2^{\mathrm{b}}$ & 1.1 & $15.5^{\mathrm{a}, \mathrm{b}}$ & 2.5 & $<0.05$ \\
\hline C-peptide, nmol/L & 1.3 & 0.1 & 1.1 & 0.1 & 1.2 & 0.1 & 1.1 & 0.1 & 0.52 \\
\hline HOMA-IR & $12.4^{\mathrm{a}}$ & 3.3 & $4.8^{b}$ & 0.9 & $4.1^{\mathrm{b}}$ & 0.5 & $7.3^{b}$ & 1.4 & $<0.05$ \\
\hline $\mathrm{HbA} 1 \mathrm{c}, \mathrm{mmol} / \mathrm{mol}$ & 73.7 & 4.5 & & & $67.6^{2}$ & 3.3 & $61.3^{2}$ & 4.0 & 0.39 \\
\hline GIP, pg/ml & 206.5 & 37.2 & 142.9 & 16.6 & 136.5 & 13.4 & & & 0.20 \\
\hline GLP-1, pM & $6.1^{\mathrm{a}}$ & 1.2 & $3.2^{\mathrm{b}}$ & 0.5 & $4.8^{\mathrm{a}, \mathrm{b}}$ & 0.7 & & & $<0.05$ \\
\hline Ghrelin, pg/ml & $341.2^{\mathrm{a}}$ & 51.0 & $651.5^{b}$ & 89.5 & $712.3^{b}$ & 95.8 & & & $<0.05$ \\
\hline
\end{tabular}

SE, standard error; HOMA-IR, homeostasis model assessment-estimated insulin resistance; GIP, gastric inhibitory peptide; GLP-1, glucagon-like peptide.

${ }^{1}$ Distinct letters indicate significant differences between time points by a one-way analysis of variance (ANOVA]) with least significant difference (LSD) post hoc test, $P<0.05$.

(In other words, the same letter shown at two different time points indicates no significant difference between those two points.)

${ }^{2}$ Indicates significant differences compared with baseline ( 0 weeks post-implant) by a paired samples $t$ test $(P<0.05)$.

within 1 week after DJBL implantation, but became even more pronounced after a prolonged post-implant period. Fat mass did not change in the first week post-implant, but started to decline in the following post-implant period, with a significant reduction from baseline after 6 months ( $\bullet$ Table 1 and $\bullet$ Fig. 2 ).

Parameters linked to glycemic control, were found to be substantially changed after DJBL implantation. Fasting insulin levels and the calculated homeostasis model assessment-estimated insulin resistance (HOMA-IR) index showed a significant decrease in the first 4 weeks post-implant. Between 4 weeks and 6 months postimplant, a subtle rise was seen for both parameters; however HOMA-IR was still significantly different from baseline levels ( Table 1 and $\bullet$ Fig. 2). For fasting glucose, a significant reduction from baseline was detected at 4 weeks post-implant, and HbA1c, which reflects the average plasma glucose concentration over a prolonged period of time, showed a continuous decline during the 6 months after DJBL implantation ( $\bullet$ Table 1 ).

As well as these changes in plasma parameters that already indicated a substantial improvement in glycemic control, we observed a drastic reduction $(\sim 2 \%)$ in overall diabetes medication after placement of the DJBL ( $\bullet$ Fig.3). Medication before and after DJBL implantation is presented in more detail in $\bullet$ Table 2, distinguishing between oral and insulin dosages. The substantial reduction in diabetes medication, seen already in the first week after implantation, emphasizes that the DJBL provokes a rapid and substantial improvement of glycemic control in obese type 2 diabetes patients.

To assess whether the improvement of glycemic control seen after DJBL implantation could be linked to the decrease in body weight, BMI, or fat mass, we performed a Pearson's correlation analysis. Therefore, we first calculated the area under curve (AUC) for the changes during the 6 months (0-24 weeks) post-implant. - Fig. 4 demonstrates that only the decrease in BMI showed a moderate positive correlation with the reduction in HbA1c levels $(r=0.662, P=0.026)$. This indicates that there might be a link between weight loss and improved glycemic control, but this relation is likely to be not very strong.
Gut hormone responses to implantation of the DJBL

The strongest effects of the DJBL were visible within the first 4 weeks post-implantation ( $\mathbf{F i g . 2}$ ). This is especially true for parameters linked to glycemic control, namely, fasting insulin and glucose and the calculated HOMA-IR. To evaluate the potential role of gut hormones in these early effects that were seen after implantation of the DJBL, we measured plasma levels of ghrelin, GIP and GLP-1, as these gut hormones are suggested to be involved in early improvement of glycemic control after RYGB surgery. Fasting plasma levels of ghrelin, GIP, and GLP-1 were analyzed before and at 1 and 4 weeks post-implantation ( $\bullet$ Fig.5). No significant changes in GIP were found, although 4 weeks after implantation a tendency for a decrease in GIP levels could be detected $(P=0.09)$. A remarkable pattern was found for the plasma GLP-1 response to DJBL implantation, as a significant GLP-1 decrease was found at 1 week post-implant with an elevation (nearly) back to baseline levels in the following 3 weeks ( $\bullet$ Fig.5). For ghrelin, a significant elevation was seen after DJBL implantation, with the highest induction in the first week post-implant ( $\bullet$ Fig.5).

A Pearson's correlation analysis using AUC data representing the changes during weeks $0-4$ post-implant ( $\bullet$ Fig.4), showed that GLP-1 responses are highly correlated to the changes in fasting insulin levels and HOMA-IR index $(r=0.728, P=0.011$ and $r=$ $0.820, P=0.004$, respectively). No correlation was found between the changes in gut hormones and the reduction in body weight, BMI and/or fat mass after placement of the DJBL. These data indicate that GLP-1 might play a role in the rapid improvement of glycemic control induced by DJBL implantation, but that this role is probably independent of weight loss and/or reduction of fat mass.

\section{Discussion}

$\nabla$

Recent studies have suggested a positive effect of RYGB surgery on remission of type 2 diabetes compared with restrictive procedures and conventional therapy, because of a direct effect on the 

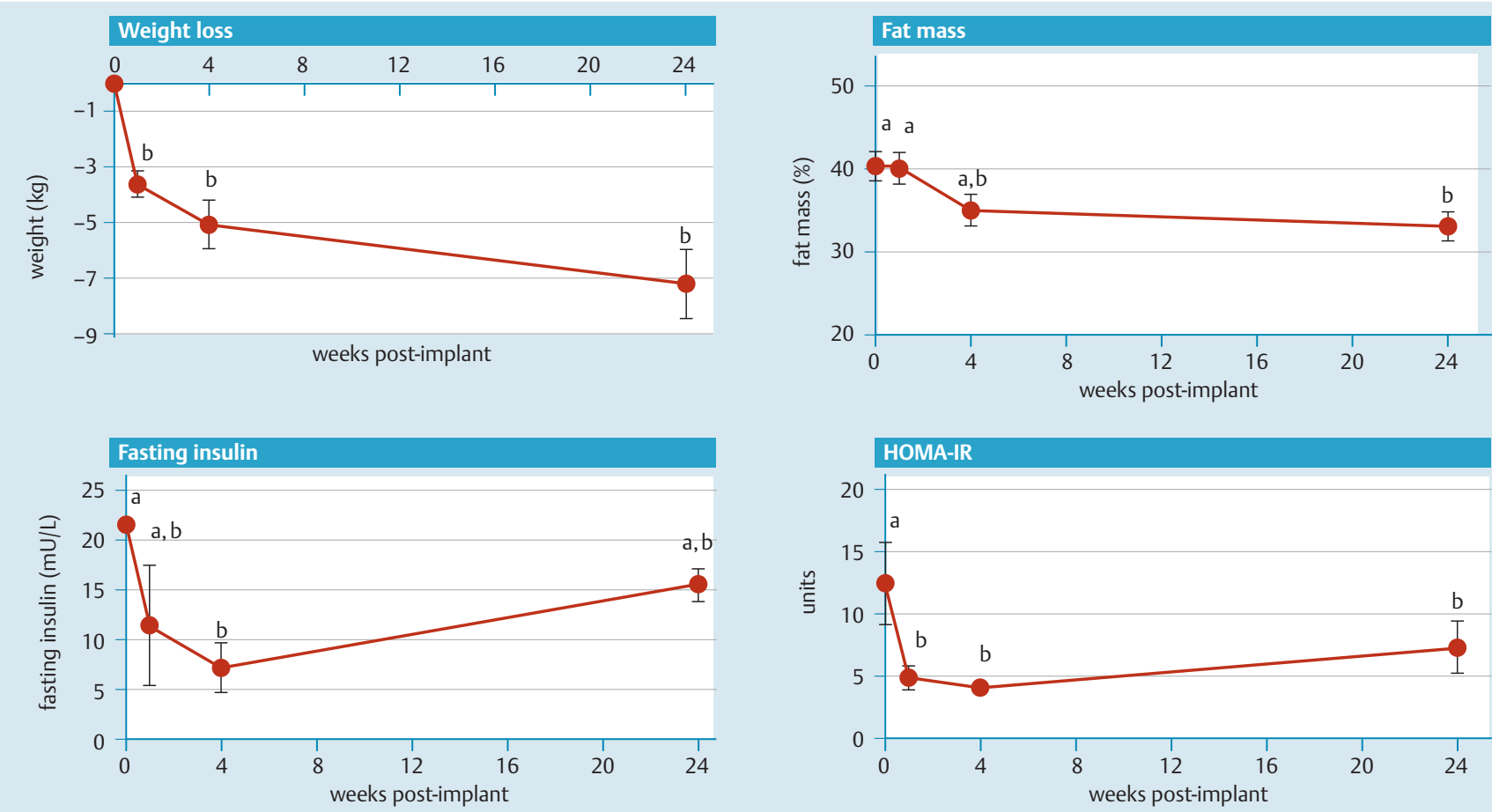

Fig. 2 Effect of duodenal-jejunal bypass liner (DJBL) implantation on weight loss, fat mass, fasting plasma insulin, and homeostasis model assessmentestimated insulin resistance (HOMA-IR) index. Weight loss, fat mass, fasting plasma insulin, and HOMA-IR index were determined pre-implant and 1, 4 and 24 weeks after DJBL implantation in all 12 patients. Data are visualized as mean ( \pm standard error [SE]). Distinct letters indicate significant differences between time points (or, the same letter shown at two different time points indicates no significant difference between those two points), $\mathrm{P}<0.05$ (one-way analysis of variance [ANOVA] with least significant difference [LSD] post hoc test).

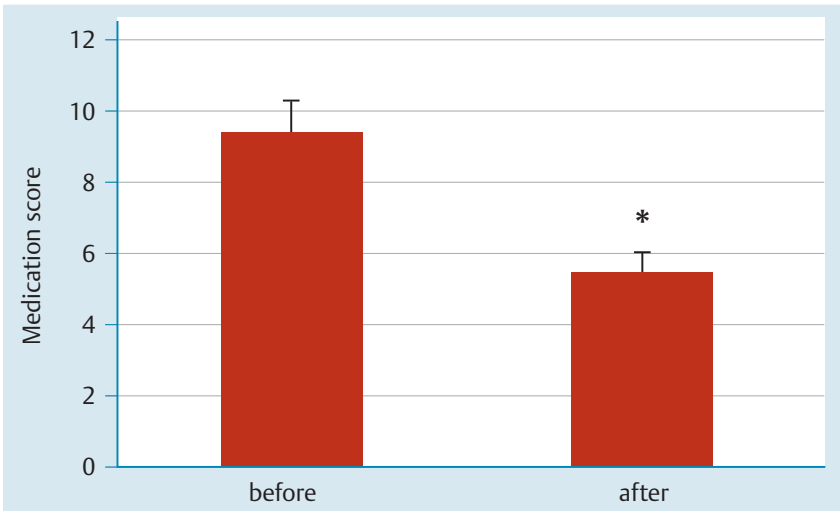

Fig. 3 Effect of duodenal-jejunal bypass liner (DJBL) implantation on diabetes medication score. Diabetes medication score was assessed for all 12 patients, before and after implantation of the DJBL. Data are visualized as mean ( \pm standard error $[\mathrm{SE}]) .{ }^{*} P<0.01$ (paired samples $t$ test).

hormonally active gut $[5,8,18]$. These early postoperative hormonal effects could be the result of the exclusion of the duodenal-jejunal part of the gut in RYGB surgery $[10,11]$. DJBL is a novel bariatric technique that mimics RYGB surgery and covers the same duodenal-jejunal part of the gut [19]. Our study shows that DJBL implantation induces a beneficial effect on remission of type 2 diabetes in obese patients, at least up till 24 weeks post-implant. So, our study indicates that DJBL implantation might be a novel and alternative bariatric procedure to induce remission of type 2 diabetes in obese patients, especially as it is a much less invasive technique. However, future clinical studies (e.g. randomized trials) are essential to directly compare and dis-
Table 2 Average medication score in 12 patients with type 2 diabetes before and after implantation of duodenal-jejunal bypass liner (DJBL). ${ }^{1}$

\begin{tabular}{|c|c|c|c|}
\hline \multicolumn{2}{|c|}{ Type 2 diabetes medication } & \multirow{2}{*}{$\begin{array}{l}\text { Average } \\
\text { score before } \\
\text { implanta- } \\
\text { tion }^{1} \\
4.4\end{array}$} & \multirow{2}{*}{$\begin{array}{l}\text { Reduction } \\
\text { after DJBL, \% } \\
\\
6\end{array}$} \\
\hline Oral & Metformin $500 \mathrm{mg}$ & & \\
\hline & Glimepiride $2 \mathrm{mg}$ & 2.9 & 57 \\
\hline & Tolbutamide $500 \mathrm{mg}$ & 4.0 & 100 \\
\hline \multirow[t]{4}{*}{ Insulin } & Insulin glargine 10 units & 4.4 & 69 \\
\hline & Insulin aspart 10 units & 6.7 & 66 \\
\hline & Exenatide 10 units & 3.0 & 100 \\
\hline & Vildagliptin $50 \mathrm{mg}$ & 1.5 & 100 \\
\hline
\end{tabular}

${ }^{1}$ Medication score calculated by adding one point for each medication unit (medication units: metformin $500 \mathrm{mg}$, glimepiride $2 \mathrm{mg}$, tolbutamide $500 \mathrm{mg}$, vildagliptin $50 \mathrm{mg}$, insulin glargine 10 units, insulin aspart 10 units, exenatide 10 units.

tinguish the effects of RYGB surgery, low calorie diets, and placement of a DJBL. These must show the additional value of DJBL implantation and prove the validity of the DJBL as a long-lasting treatment for the obese diabetic patient.

In the study of de Jonge et al. changes in postprandial responses of GLP-1 and GIP were reported after placement of a DJBL [20]. These changes, and especially the increase in GLP-1 postprandial response, are highly similar to changes induced by RYGB surgery that are suggested to contribute to the early remission of type 2 diabetes [8]. Fasting GLP-1 and GIP levels in our study are more difficult to directly link to early remission of type 2 diabetes and more likely reflect the effect of caloric restriction that is induced 


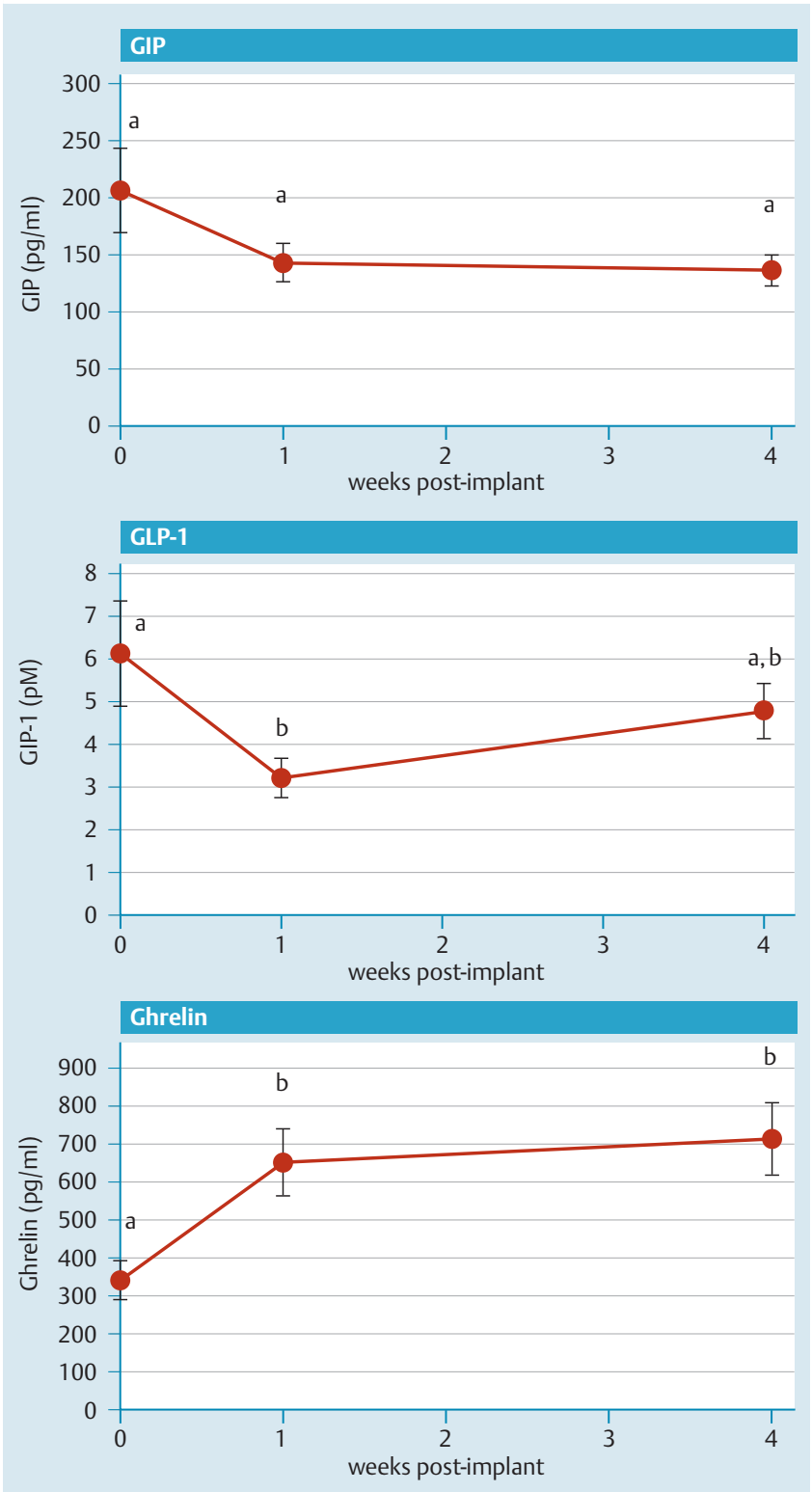

Fig. 4 Responses of gut hormones ghrelin, gastric inhibitory peptide (GIP), and glucagon-like peptide (GLP-1) after implantation of the duodenal-jejunal bypass liner (DJBL). Fasting plasma levels of GIP GLP-1 and ghrelin were determined before (day 0 ) and at 1 and 4 weeks after implantation of the DJBL in all 12 patients. Data are visualized as mean ( \pm standard error [SE]). Distinct letters indicate significant differences between time points (or, the same letter shown at two different time points indicates no significant difference between those two points), $P<0.05$ (one-way analysis of variance $[A N O V A]$ with least significant difference [LSD] post hoc test).

by placement of the DJBL. For instance, the early "dip" in GLP-1 levels at 1 week post-implant could be explained by the consumption of liquid and ground/puréed food in the first week post-implant. This food is probably more easily absorbed in the proximal gut and will therefore hardly reach the distal small intestine where GLP-1 is mainly expressed. Even though these changes in fasting hormone levels are expected to be normal physiological responses to restriction and deprivation of diet, fasting GLP-1 shows a significant correlation with fasting insulin and HOMA-IR, indicating that also the changes in fasting GLP-1 might play a role in improvement of glycemic control.
Surprisingly, we found an increase in ghrelin levels after placement of the DJBL, whereas mostly a decrease in fasting ghrelin levels is reported after RYGB surgery. Ghrelin is often suggested to be a "foregut factor" that plays an important role in type 2 diabetes remission after RYGB surgery [8,11]. Decreased ghrelin levels would lower the levels of growth hormone, cortisol, and epinephrine, hormones that are known to counter-regulate insulin action. Moreover, decreased ghrelin levels are supposed to stimulate secretion of the insulin-sensitizing hormone adiponectin and might have a direct effect on insulin secretion by reduced binding to the ghrelin-receptor expressed in the pancreatic islets. Our study, however, does not support this potential role of ghrelin in improvement of glycemic control, as we found an elevation of ghrelin, but still a substantial remission of type 2 diabetes. The elevation of ghrelin that we found could be explained by the reduced food intake that is prescribed by the dietary guidelines that accompany the placement of the DJBL. Ghrelin is a well-known "hunger signal" that is predominantly secreted by the stomach and under normal physiological conditions, a reduced consumption of nutrients leads to an increase in plasma ghrelin levels [21]. The reduced ghrelin levels that are often seen after RYGB surgery might be explained by a surgically induced disruption of the vagal input to a majority of ghrelin-producing cells during creation of the gastric pouch and bypass of the gastric fundus. Blockade of vagal impulses has been reported to reduce circulation ghrelin levels $[21,22]$. Our data indicate that these reduced ghrelin levels after RYGB surgery have probably no or minimal effect on remission of type 2 diabetes. Remarkably, despite the rise in levels of the "hunger hormone" ghrelin after implantation of the DJBL, patients also reported a reduced feeling of hunger (nonvalidated subjective observation) as is seen after RYGB surgery. So, altogether, we conclude that the role of ghrelin in hunger sensation and remission of type 2 diabetes after bariatric surgery remains unclear. Moreover, our data suggest that other "foregut factors" than ghrelin must be involved in these processes.

In summary, implantation of a DJBL results in significant weight loss and a rapid and substantial improvement of glycemic control. As for gut hormone responses, in contrast to RYGB surgery, implantation of the DJBL seems to preserve normal physiological responses of gut hormones linked to nutrient deprivation and dietary restriction, as elevated levels of ghrelin were found after reduced calorie intake and a "dip" in GLP-1 levels was found after temporary consumption of ground/puréed food. Most interestingly, our study provides indications that the reduced ghrelin levels that are found after RYGB surgery cannot be a contributing "foregut factor" in the early improvement of glycemic control. This is an intriguing finding, which must be further explored in future research.

\section{Funding}

This research did not receive any specific grant from any funding agency in the public, commercial or not-for-profit sector.

\section{Author contributions}

P. K. contributed to study design, performed clinical procedures, researched data and wrote the manuscript. K. D. was involved in clinical procedures and reviewed the manuscript. F. B. contributed to study design, was involved in clinical procedures and reviewed the manuscript. I. J. contributed to study design, was involved in clinical procedures and reviewed/edited the manuscript. P. W. was involved in clinical procedures and reviewed the manuscript. M. G. was involved in clinical procedures and re- 


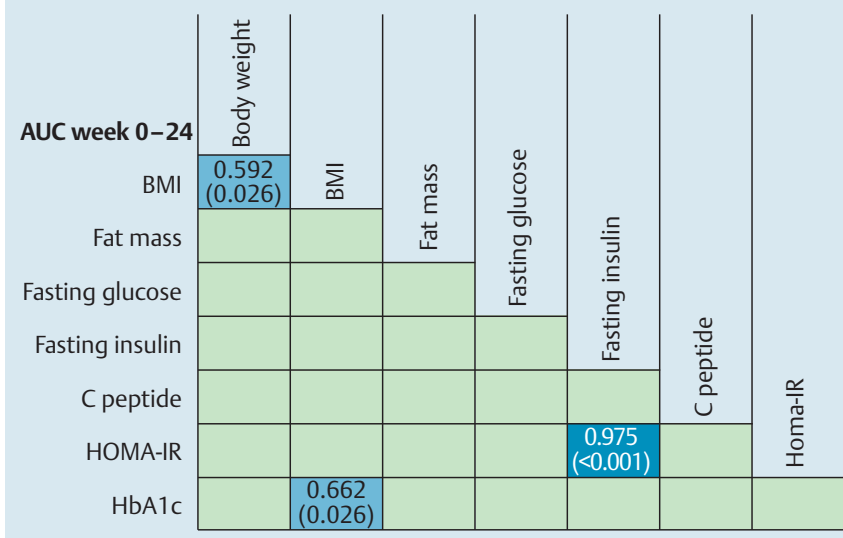

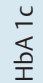

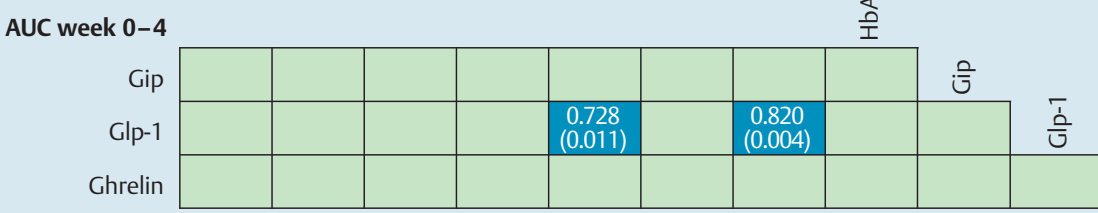

Fig. 5 Pearson correlation analysis using AUC data For body weight, BMI, fat mass, fasting glucose, fasting insulin, C-peptide, HOMA-IR and HbA1c, Pearson correlation analysis was performed using AUC data of $0-24$ weeks after DJBL implantation. For gut hormones GIP, GLP-1 and ghrelin AUC data of $0-4$ weeks post-implant were used. Grenn boxes indicate moderate correlation, blue boxes indicate a high correlation. Empty boxes mean no significant correlation was found. AUC, area under curve; BMI, body mass index; HOMA-IR, homeostasis model assessment-estimated insulin resistance; Gip, gastric inhibitory peptide; Glp-1, glucagon-like peptide.

viewed the manuscript. M. M. reviewed/edited the manuscript. N. d. W. contributed to study design, performed analytical procedures, researched data, and wrote the manuscript.

Competing interests: P. Koehestanie, I. M. Janssen, K. Dogan and P. J. Wahab disclose the following financial relationships relevant to this publication: They all received consultancy fees from GI dynamics for proctoring. Other authors have no conflicts of interest relevant to this article.

\section{Acknowledgments}

The authors would like to thank the patients for contributing to this study.

\section{References}

1 Flegal KM, Carroll MD, Ogden CL et al. Prevalence and trends in obesity among US adults, 1999-2008. JAMA 2010; 303: 235-241 PubMed

2 Wild S, Roglic G, Green A et al. Global prevalence of diabetes: estimates for the year 2000 and projections for 2030. Diabetes Care 2004; 27: $051047-1053$

3 Kelly T, Yang W, Chen CS et al. Global burden of obesity in 2005 and projections to 2030. Int J Obes (Lond) 2008; 32: 1431 - 1437

4 Björntorp P. Results of conservative therapy of obesity: correlation with adipose tissue morphology. Am J Clin Nutr 1980; 33: 370 - 375

5 Buchwald $H$, Avidor Y, Braunwald E et al. Bariatric surgery: a systematic review and meta-analysis. JAMA 2004; 292: 1724-1737

6 Schauer PR, Burguera B, Ikramuddin S et al. Effect of laparoscopic Rouxen Y gastric bypass on type 2 diabetes mellitus. Ann Surg 2003; 238 467-484 (discussion 84-85)

7 Beckman LM, Beckman TR, Sibley SD et al. Changes in gastrointestinal hormones and leptin after Roux-en-Y gastric bypass surgery. JPEN J Parenter Enteral Nutr 2011; 35: 169-180

8 Hage MP, Safadi B, Salti I et al. Role of gut-related peptides and other hormones in the amelioration of type 2 diabetes after Roux-en-Y gastric bypass Surgery. ISRN Endocrinol 2012; 2012: 504756

9 Falkén Y, Hellström PM, Holst JJ et al. Changes in glucose homeostasis after Roux-en-Y gastric bypass surgery for obesity at day three, two months, and one year after surgery: role of gut peptides. J Clin Endocrinol Metab 2011; 96: 2227-2235
10 Rubino F, Forgione A, Cummings DE et al. The mechanism of diabetes control after gastrointestinal bypass surgery reveals a role of the proximal small intestine in the pathophysiology of type 2 diabetes. Ann Surg 2006; 244: 741 - 749

11 Thaler JP, Cummings DE. Minireview: Hormonal and metabolic mechanisms of diabetes remission after gastrointestinal surgery. Endocrinology 2009; 150: $2518-2525$

12 Peterli R, Steinert RE, Woelnerhanssen B et al. Metabolic and hormona changes after laparoscopic Roux-en-Y gastric bypass and sleeve gastrectomy: a randomized, prospective trial. Obes Surg 2012; 22: 740 748

13 Schouten R, Rijs CS, Bouvy ND et al. A multicenter, randomized efficacy study of the EndoBarrier Gastrointestinal Liner for presurgical weight loss prior to bariatric surgery. Ann Surg 2010; 251: 236-243

14 de Moura EGH, Orso IRB, da Costa Martins B et al. Improvement of insulin resistance and reduction of cardiovascular risk among obese patients with type 2 diabetes with the duodenojejunal bypass liner Obes Surg 2011; 21: $941-947$

15 de Moura EGH, Martins BC, Lopes GS et al. Metabolic improvements in obese type 2 diabetes subjects implanted for 1 year with an endoscopically deployed duodenal-jejunal bypass liner. Diabetes Technol Ther 2012; 14: 183-189

16 Escalona A, Pimentel F, Sharp A et al. Weight loss and metabolic improvement in morbidly obese subjects implanted for 1 year with an endoscopic duodenal-jejunal bypass liner. Ann Surg 2012; 255: $1080-1085$

17 Dorman RB, Serrot FJ, Miller CJ et al. Case-matched outcomes in bariatric surgery for treatment of type 2 diabetes in the morbidly obese patient. Ann Surg 2012; 255: 287-293

18 Mingrone G, Panunzi S, De Gaetano A et al. Bariatric surgery versus conventional medical therapy for type 2 diabetes. N Engl J Med 2012; 366 : $1577-1585$

19 Zhu L, Mo Z, Yang X et al. Effect of laparoscopic Roux-en-Y gastroenterostomy with $\mathrm{BMI}<35 \mathrm{~kg} / \mathrm{m}(2)$ in type 2 diabetes mellitus. Obes Surg 2012; 22: $1562-1567$

20 de Jonge C, Rensen SS, Verdam FJ et al. Endoscopic duodenal-jejunal bypass liner rapidly improves type 2 diabetes. Obes Surg 2013; 23 : $1354-1360$

21 Cummings DE, Shannon MH. Ghrelin and gastric bypass: is there a hormonal contribution to surgical weight loss? J Clin Endocrinol Metab 2003; 88: 2999-3002

22 Sundbom M, Holdstock C, Engström BE et al. Early changes in ghrelin following Roux-en-Y gastric bypass: influence of vagal nerve functionality? Obes Surg 2007; 17: 304-310 